\title{
Wiping out MRSA: effect of introducing a universal disinfection wipe in a large UK teaching hospital
}

\author{
Mark I. Garvey ${ }^{1,2^{*}}$ (D, Martyn A. C. Wilkinson ${ }^{1}$, Craig W. Bradley ${ }^{3}$, Kerry L. Holden ${ }^{3}$ and Elisabeth Holden ${ }^{1}$
}

\begin{abstract}
Background: Contamination of the inanimate environment around patients constitutes an important reservoir of MRSA. Here we describe the effect of introducing a universal disinfection wipe in all wards on the rates of MRSA acquisitions and bacteraemias across a large UK teaching hospital.

Methods: A segmented Poisson regression model was used to detect any significant changes in the monthly numbers per 100,000 bed days of MRSA acquisitions and bacteraemias from April 2013 - December 2017 across QEHB.

Results: From April 2013 to April 2016, cleaning of ward areas and multi-use patient equipment by nursing staff consisted of a two-wipe system. Firstly, a detergent wipe was used, which was followed by a disinfection step using an alcohol wipe. In May 2016, QEHB discontinued the use of a two-wipe system for cleaning and changed to a one wipe system utilising a combined cleaning and disinfection wipe containing a quaternary ammonium compound. The segmented Poisson regression model demonstrated that the rate of MRSA acquisition/100,000 patient bed days was affected by the introduction of the new wiping regime (20.7 to 9.4 per 100,000 patient bed days; $p<0.005$ ).

Discussion: Using a Poisson model we demonstrated that the average hospital acquisition rate of MRSA/ 100,000 patient bed days reduced by $6.3 \%$ per month after the introduction of the new universal wipe.

Conclusion: We suggest that using a simple one wipe system for nurse cleaning is an effective strategy to reduce the spread and incidence of healthcare associated MRSA.
\end{abstract}

Keywords: Meticillin-resistant Staphylococcus aureus, MRSA bacteraemias, MRSA acquisitions, Disinfection wipes

\section{Introduction}

Staphylococcus aureus is a major cause of healthcare-associated infection worldwide [1-3]. Meticillin-resistant S. aureus (MRSA) has become prevalent in most parts of the world [1-4]. Despite its decline in incidence in several European countries, MRSA infection remains a major cause of avoidable morbidity and mortality in patients admitted to hospital [1-4]. It results in increased length of hospital stay, risk of death and treatment costs [4], with colonized and infected patients acting as reservoirs for the spread of MRSA within hospitals [5, 6].

\footnotetext{
* Correspondence: mark.garvey@uhb.nhs.uk; m.i.garvey@bham.ac.uk 'University Hospitals Birmingham NHS Foundation Trust, Queen Elizabeth Hospital Birmingham, Edgbaston, Birmingham B15 2WB, England ${ }^{2}$ Institute of Microbiology and Infection, The University of Birmingham, Edgbaston, Birmingham, England

Full list of author information is available at the end of the article
}

Isolation and decolonization are the two main targeted control measures for reducing transmission of MRSA within hospitals $[7,8]$.

It is acknowledged that the healthcare environment plays a key role in facilitating the transmission of important pathogens responsible for healthcare-associated infections [9]. These pathogens include vancomycin-resistant enterococci (VRE), Clostridium difficile and MRSA [9]. Such organisms are able to survive in the environment for many days, posing an ongoing risk of transmission and acquisition by hospital patients [10]. In recent years, there has been more interest from infection control staff, clinicians, health planners and government on maintaining a clean environment [9-11]. Contamination of the inanimate environment around patients constitutes an important reservoir of multi drug

(c) The Author(s). 2018 Open Access This article is distributed under the terms of the Creative Commons Attribution 4.0 International License (http://creativecommons.org/licenses/by/4.0/), which permits unrestricted use, distribution, and 
resistant organisms, with the risk of healthcare-associated infection increasing significantly if the patient previously occupying the room had MRSA, VRE, C. difficile or other multidrug resistant pathogen [11]. Patients in rooms previously occupied by MRSA colonized or infected patients had a twofold increased risk of MRSA acquisition [11, 12]. Garvey et al., (2017) found that during a PVL positive MRSA outbreak, up to $40 \%$ of the environmental areas on a ward sampled contained MRSA [13]. Even with enhanced cleaning, the PVL positive MRSA strain was not eliminated from the environment [13]. It is not uncommon for hand hygiene and the environment to play a role in the transmission of MRSA in a hospital setting; various reports have previously detailed these transmission routes $[12,14]$.

Between April 2013 to April 2016, ward areas at Queen Elizabeth Hospitals Birmingham (QEHB) part of University hospitals Birmingham were cleaned by nursing staff using a two-wipe system. Specifically, this included routine cleans of the ward environment, patient equipment and post discharge cleaning of bed spaces and beds of patients without known nosocomial pathogens. This entailed using, firstly a detergent wipe, followed by a disinfection step using an alcohol wipe. From May 2016, QEHB discontinued the two-wipe system, changing to a one-wipe system utilising a combined cleaning and disinfection wipe. We report the effect of introducing a universal disinfection wipe in all wards on the rates of MRSA acquisition across QEHB.

\section{Materials and methods \\ Setting}

Queen Elizabeth Hospital Birmingham (QEHB), part of University Hospitals Birmingham (UHB) NHS Foundation Trust is a tertiary referral teaching hospital in Birmingham, UK that provides clinical services to nearly one million patients every year [8].

\section{MRSA screening}

MRSA screening of all emergency, elective surgical patients and day case patients (deemed to have high risk procedures such as surgery) admitted to UHB is standard practice. Only day case patients whom receive minor procedures such as endosocpy are not screened. Swabs are taken from the nose, groin and throat as well as any wounds or sites of invasive devices. Inpatients with greater than 28 days' stay are rescreened every 4 weeks. Only one change to MRSA screening has been undertaken at QEHB during this report, in August 2014 MRSA screening to low risk patients such as day-case patients was stopped.

\section{MRSA acquisitions}

At QEHB a patient is defined as acquiring MRSA if they have a negative admission screen and then have MRSA isolated from a subsequent screen or clinical specimen, 48 hours after admission. Only MRSA acquisitions at QEHB were included in the analysis. All MRSA acquisitions are identified as previously described by Garvey et al., (2016) [14, 15].

\section{MRSA bacteraemia}

All MRSA bacteraemias were attributed as healthcare associated or non-healthcare associated using the criteria of the Centres for Disease Control and Prevention/National Healthcare Safety Network for national reporting purposes [16]. All bacteraemias then go through a Post Infection Review process for assignment of apportionment either to the acute Hospital, Clinical Commissioning Group (CCG, community based) or 'third party', regardless of attribution to healthcare or non-healthcare associated [16]. The third part of the amendment allows for the assignment of a case of MRSA bloodstream infection to a 'third party' through the arbitration process lead by the Regional Director of Nursing or Regional Medical Director. Third party assignment provides an acknowledgement of the complex nature of MRSA bloodstream infections being reported which previously may have been allocated by default to acute hospitals or CCGs who were not involved in the patients care or who can provide a strong case following the Post Infection Review that there were no possible failings in patient care. Only bacteraemias that were assigned to the acute hospital (QEHB) were included in the analysis [16]. Similar to acquisitions, all MRSA bacteraemias are molecularly typed to help identify transmission links $[14,15]$.

\section{Current cleaning programme}

The hospital utilises an environmental cleaning procedure consisting of four different grades. Red and amber cleans are employed when the prior occupant of the room or bed space was known or suspected of having an alert organism or diarrhoea. An alert organism is classified as a transmissible nosocomial pathogen including, but not limited to: $C$. difficile, norovirus, MRSA, extended spectrum $\beta$-lactamase (ESBL) producing organisms, VRE, Influenza, Mycobacterium tuberculosis and Streptococcus pyogenes. The cleaning methods for these grades require additional training for the operators; for example, housekeeping assistants receive training for curtain changes, use of a hydrogen peroxide misting system, cleaning of sinks and making up the chemicals. Included in both red and amber cleans, are a surface and equipment clean using a chlorine- and detergent-based agent (ChlorClean', Guest Medical Limited, Edenbridge, UK) with disposable cloths, and the curtains are changed. In addition, the red clean involves the application of $6 \% \mathrm{w} / \mathrm{v}$ hydrogen peroxide misting by aerosolisation (Oxyfarm, Glasgow, UK). For cleans where the patient 
was not known or suspected of harbouring an alert organism, a green clean is carried out using a single detergent/disinfectant wipe (wipe A - Table 1). Nursing staff are responsible for green cleans, which include a surface and equipment clean using a single detergent/disinfectant wipe (wipe A - Table 1). All bed-side equipment are wiped until visibly-clean, as well as the bed frame, touch points including the nurse-call handset, door handles and bed-side chair. The curtains are inspected for signs of soiling and are changed if required. Nurses have been trained in how to store and use the universal wipes and regularly use a new wipe when, either the existing wipe is dry, or moving on to a new piece of equipment.

\section{Cleaning programme pre-May 2016}

Compared to the current protocol, the only difference in cleaning involved the green clean, where two wipes were used instead of one. From April 2013 to April 2016, the cleaning of ward areas by nursing staff involved firstly, a detergent wipe, followed by a disinfection step using an alcohol wipe (wipes B and C, respectively - Table 1). Post May 2016 QEHB moved away from a two-wipe system to a universal one wipe system. The decision to choose wipe A was based on the ease of us, log reduction killing against a range of nosocomial microorganisms and staff preference (data not shown). Before implementation and hospital wide use of wipe A, several wipes were tested for efficacy in cleaning against a range of nosocomial organisms with the most efficacious (top two wipes) at reducing the number of nosocomial microorganisms being trialled on two wards for staff feedback. The wipe most preferred by staff based on a questionnaire was then chosen for use across QEHB.

\section{Education}

Before the implementation of the new single disinfectant wipe (wipe A), an education package spanning one month was implemented throughout QEHB. The training package was delivered by trained Infection Control

Table 1 Wipes used at QEHB between April 13 to December 17 including chemical composition of wipes

\begin{tabular}{|c|c|c|}
\hline Wipe & $\begin{array}{l}\text { Detergent/ disinfectant } \\
\text { wipe }\end{array}$ & Contents \\
\hline$A$ & $\begin{array}{l}\text { Detergent/ disinfectant } \\
\text { wipe (Clinell Universal } \\
\text { Sanitising Wipes, GAMA } \\
\text { Healthcare Limited, UK) }\end{array}$ & $\begin{array}{l}\text { Bezalkonium chloride } \leq 0.5 \% \text {, } \\
\text { Didecyl dimethyl ammonium } \\
\text { chloride } \leq 0.5 \% \text {, Polyhexamethylene } \\
\text { biguanide (PHMB) } \leq 0.10 \% \text {, } \\
\text { Water }>75 \% \text {, Additives each }<1 \%\end{array}$ \\
\hline B & Detergent wipe & $\begin{array}{l}\text { Phenoxyethanol }<1 \% \text {, Alkyl } \\
\text { polyglycoside }<0.2 \%, \\
\text { Diethylene glycol }<0.1 \% \text {, } \\
\text { 2-Octyl-2H-Isothiazol-3-one } \\
<0.01 \%\end{array}$ \\
\hline C & Alcohol wipe & Propan-2-ol 50-80\% \\
\hline
\end{tabular}

Nurses from GAMA healthcare (GAMA healthcare, UK) and involved teaching ward nurses the following: how to use the universal wipe for a surface and equipment clean; equipment to be cleaned, including all bed-side equipment, the bed frame, touch points including the nurse-call handset, door handles and bed-side chair. Nurses were also trained how to store and use the universal wipes, and to use a new wipe when either the old wipe became dry or moving on to a new piece of equipment. This practice was audited by Infection Prevention and Control Nurses. Between April 2016 - December 20171160 frontline healthcare workers primarily nurses were trained in how to use the new wipe.

\section{Audits}

Regular audits were undertaken including monitoring hand hygiene compliance, monitoring the appropriate use of personal protective equipment and monitoring environmental cleanliness. Hand hygiene audits are undertaken by the wards either daily or weekly and validated by the Infection prevention and Control Team. PPE audits are undertaken monthly by the Infection prevention and control team. Environmental cleanliness audits are undertaken monthly by the facilities/estates team and validated by the Infection prevention and control Team.

\section{ICU decolonization timeline}

As described previously by Bradley et al., (2017) before August 2014 all ICU patients at UHB received universal MRSA decolonization therapy regardless of their MRSA status [8]. Between August 2014-December 2015 universal decolonization therapy of all ICU patients was withdrawn and only MRSA positive patients received MRSA decolonization therapy [8]. In December 2015 UHB reintroduced universal MRSA decolonization therapy to all ICU patients regardless of their MRSA status [8].

\section{Statistical analyses}

Segmented Poisson regression models containing offsets for patient bed days were used to detect any significant changes in the rate of MRSA acquisitions and bacteraemias from April 2013 - December 2017. There were no other changes to the Infection Prevention and Control policy or practice during this period. 'Full' models were constructed containing terms for changes in level and gradient associated with the following potentially-important interventions: the withdrawal and reintroduction of universal decolonization of patients in the intensive care unit and the change in wiping regime. Backward step-wise regression was performed on the 'full' Poisson models, with the Akaike Information Criterion used to select the models that best fit the data, whilst guarding against 
over-fitting. All statistical analyses were performed using $\mathrm{R}$ version 3.4.3 [17, 18].

\section{Results \\ MRSA acquisitions}

We analysed QEHBs acquisition rate of MRSA per 100,000 patient bed days using a segmented Poisson regression model. The number of MRSA acquisitions across QEHB between April 2013 and April 2016 averaged at around 20.7 per 100,000 patient bed days (Table 2). Following the introduction of a one wipe system the number of MRSA acquisitions across QEHB decreased from 20.7 to 9.4 per 100,000 patient bed days (Table 2 and Figure 1a; 310 vs 93 acquisitions). The optimum Poisson model demonstrates that the rate of acquisition of MRSA per 100,000 patient bed days was affected by the withdrawal of universal decolonization $(\mathrm{p}=0.057)$ and by the introduction of a new one wipe regime $\left(\mathrm{p}=2.89 \times 10^{-8}\right)$. The evidence for the effect of the new wiping regime was much stronger. The model demonstrates that the average trust acquisition rate of MRSA per 100,000 patient bed days reduced by $6.3 \%$ per month after the introduction of the new wipes (Fig. 1a).

\section{MRSA bacteraemia}

The MRSA bacteraemia rate per 100,000 patient bed days for the same period was also analysed using a segmented Poisson regression model. The model demonstrated that there was a $270 \%$ increase in the average MRSA bacteraemia rate per 100,000 patient bed days during the period of cessation of universal decolonization $(p=0.00203$; Fig. $1 b)$. The model demonstrated no change in the rate of MRSA bacteraemias associated with introducing a new one wipe regime.

\section{Auditing}

Audits revealed no significant changes in hand hygiene compliance; appropriate use of personal protective equipment (PPE) or environmental cleanliness during the study (data not shown). Any failures in practice education sessions are undertaken to address any issues. MRSA screening for emergency and elective patients averages at $90 \%$ and $92 \%$ respectively, over the past five

Table 2 Total number of patients admitted to ICU and to the hospital during the 3 study periods. The total number of MRSA acquisitions and bacteraemias across QEHB are shown during the 3 study periods

\begin{tabular}{llll}
\hline & $\begin{array}{l}\text { April 13 - } \\
\text { August 14 }\end{array}$ & $\begin{array}{l}\text { August 14 - } \\
\text { March 16 }\end{array}$ & $\begin{array}{l}\text { April 16 - } \\
\text { December 17 }\end{array}$ \\
\hline Bed days - Critical Care & 35,595 & 40,165 & 51126 \\
Bed days - QEHB Total & 550,107 & 606,820 & 989,724 \\
MRSA acquisitions & 119 & 191 & 93 \\
MRSA bacteraemias & 5 & 16 & 2 \\
\hline
\end{tabular}

years at QEHB. At QEHB there is electronic prescribing so all patients who are MRSA positive receive decolonization therapy, thereby no prescribing patterns were altered during this study.

\section{Discussion}

The English Department of Health (DoH) introduced universal mandatory MRSA screening of all elective and emergency admissions to English hospitals in 2010 [19]. In 2012 the DoH commissioned a national audit (National One Week Prevalence Audit of MRSA) to review implementation and impact of patient management from universal MRSA screening [20]. The audit found implementation of universal screening was poor, MRSA admission prevalence (new cases) was low and suggested UK hospitals should seek to improve implementation of current MRSA screening policy [20]. The report made recommendations to stop routine screening in large teaching Hospitals [20]. In response to the National One Week Prevalence Audit of MRSA, QEHB implemented a revised strategy for the control of MRSA [20]. Before 2014 all ICU patients at QEHB received universal MRSA decolonization therapy regardless of their MRSA status [8]. In August 2014 QEHB discontinued the use of universal decolonization in the ICU and stopped MRSA screening to low-risk patients such as day cases having minor procedures such as endoscopy [8]. A breakpoint model identified an increase in MRSA bacteraemias and acquisitions across QEHB, subsequent to the withdrawal of universal decolonization [8]. As a result of increased rates of MRSA, universal decolonization was reintroduced into the ICU resulting in a reduction of MRSA bacteriaemias and acquisitions across QEHB [8]. Here we follow on from this work and detail the effect of introducing a universal detergent/disinfection wipe for nurse-led cleans in all wards on the rates of MRSA acquisitions across QEHB.

A segmented Poisson regression model suggests that the rate of acquisition of MRSA per 100,000 patient bed days was affected by the withdrawal of universal decolonization, as per the work by Bradley et al., (2017), and unexpectantly by the introduction of a new wiping regime [8]. Whilst the evidence for the effect of universal decolonization on the number of MRSA acquisitions was statistically rather weak, the optimum model suggested that the average rate of acquisition of MRSA per 100,000 patient bed days increased by $23 \%$ during the period of withdrawal of MRSA decolonization on ICU. The evidence for the effect of the new universal wipe regime was much stronger. The data suggest the use of a one wipe regime is associated with reducing the incidence of healthcare associated MRSA. It is not surprising that cleaning and disinfection could be so important in the reduction of MRSA acquisitions across QEHB. 


\section{a MRSA acquisition rate with segmented Poisson regression model.}

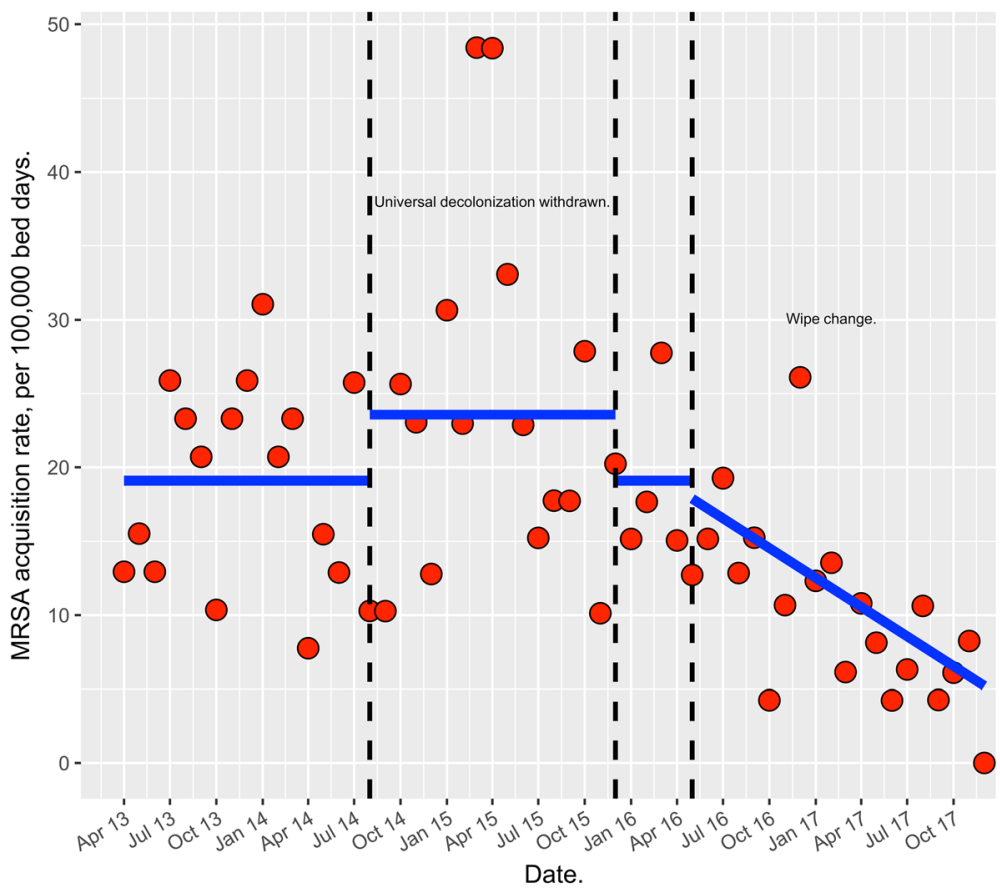

b MRSA bacteraemia rate with segmented Poisson regression model.

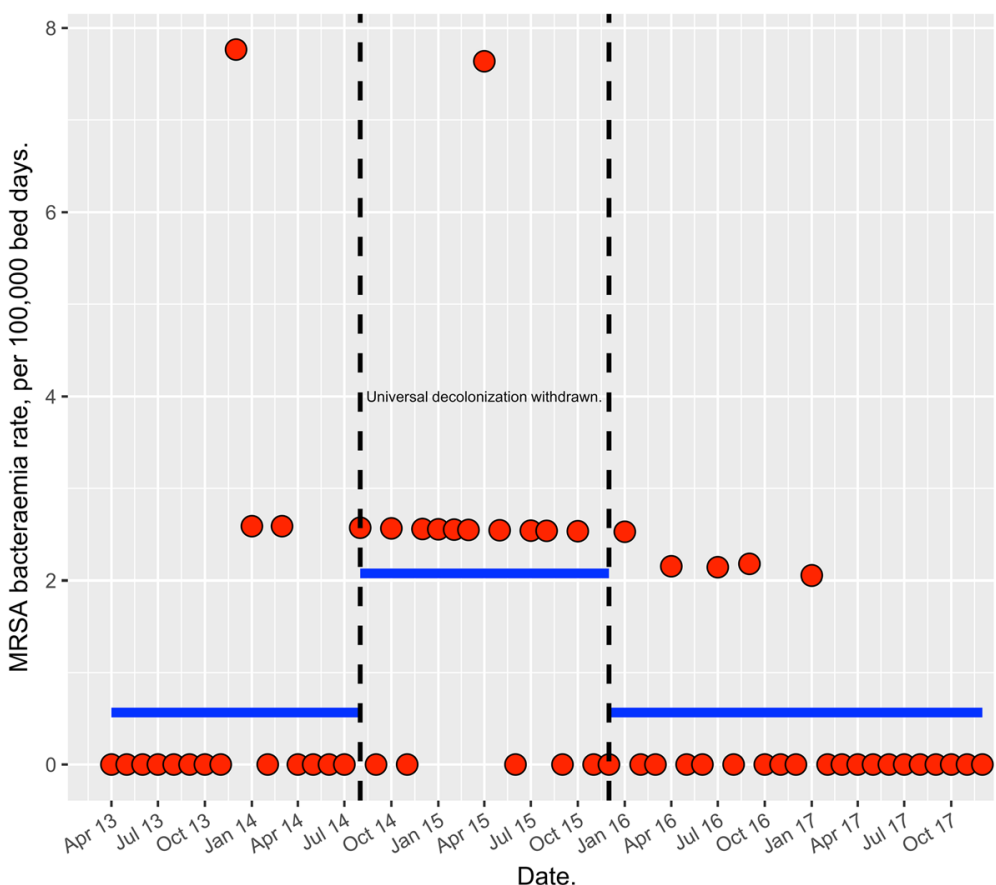

Fig. 1 Using a segmented Poisson regression model changes in hospital wide monthly MRSA acquisition rates (a) and MRSA bacteraemia rates (b) per 100,000 bed days between April 2013-December 2017. Key: A two wipe regime for nurse led cleans was undertaken at QEHB between April 2013 to April 2016; a one wipe regime for nurse led cleans was undertaken at QEHB between May 2016 to December 2017; the removal of universal MRSA decolonization therapy in the ICU at QEHB occurred during August 2014-December 2016; the reintroduction of universal MRSA decolonization therapy in the ICU at QEHB occurred between January-December 2017. The dotted lines represent the infection prevention and control interventions. The blue lines represent the mean values predicted by the Poisson regression model 
MRSA has been demonstrated to exist in the healthcare environment and cleaning has been shown to reduce the levels of MRSA in the environment [12, 13]. A systematic review by Dancer et al., (2012) revealed that MRSA is found in the healthcare environment and reductions in the environmental burden have been shown through detergent and disinfectant regimes [9]. The use of disinfectant wipes is common practice within UK hospitals for the decontamination of environmental surfaces coming into contact with patients, for example common hand touch point areas, as well as patient equipment $[21,22]$. This is often undertaken by nursing staff, with more specialised room cleans for infected patients undertaken by dedicated cleaning teams [21, 22]. This is true of the cleaning practice at QEHB, where cleans of rooms for non-infected patients are undertaken by nursing staff, predominantly using disinfectant wipes. Kundrapu et al., (2012) demonstrated that the decontamination of environmental surfaces can reduce the risk of contamination of the hands of healthcare personnel [23]. A cleaner environment including patient equipment will result in lower levels of MRSA being transferred to healthcare workers' hands and subsequently to patients. Further work is needed to elucidate whether the new wipe regime reduced the amount of environmental contamination with MRSA; environmental monitoring before and after implementation of the new wipe regime could have been one of the ways to observe this. The effect of the new wipe regime on the rate of MRSA acquisitions was an unexpected finding, further work is needed to identify if the implementation of a new wipe had an effect on the rate of patient acquisitions of other nosocomial alert organisms.

The optimum Poisson model provided clear evidence that the rate of MRSA bacteraemia per 100,000 patient bed days was affected by the withdrawal of universal decolonization as previously described by Bradley et al., (2017) [8]. The number of MRSA bacteraemias was unaffected by the change in wipe regime. The authors hypothesise the patients most at risk of developing an MRSA bacteraemia are patients which pass through the ICU. The percentage of inpatients passing through the 100 bedded ICU at QEHB is $5-10 \%$ and account for $5.5 \%$ of the total bed days. By decolonising this group of patients, the authors believe this resulted in the reduction of MRSA bacteraemias independent of the change in wipe regime.

Detergent wipes are formulated to remove contamination from surfaces (i.e. to physically clean) [24-26]. Disinfectant wipes contain specific antimicrobial agent(s) to inactivate the bioburden on surfaces, which may contain infectious microorganisms and bodily fluids [24-26]. Due to the variety of detergent and disinfectant wipes available between April 2013 and April 2016, guidance by the Royal College of Nursing dictated that the manufacturer's instructions were followed when using wipes [21, 22, 24-26]. This often entailed a detergent wipe to be used to clean the surface of gross debris/ heavy soil, followed by the use of a disinfectant wipe to disinfect the surface [21, 22]. A number of studies have advocated a ' 1 wipe, 1 surface, 1 direction' approach, which is considered to be applicable for use in practice [21, 22]. Rahm et al., (2015) demonstrated that no detergent wipe removes all contamination and if used incorrectly, contamination can be transferred to subsequent surfaces [25]. Between April 2013 and April 2016, QEHB used a two-wipe system involving a detergent wipe (wipe B) and an alcohol disinfectant wipe (wipe C). In April 2016 QEHB changed to a one wipe detergent and disinfection wipe (wipe A) for ease of use. Following the introduction, the number of MRSA acquisitions across UHB decreased from 20.7 to 9.4 per 100,000 patient bed days. It is not surprising moving from a two-wipe system to a one wipe system would affect the cleanliness of the hospital environment. Borg (2014) described infection control as a behavioural science, and here we show a change in practice to something which is simpler can have a result [27]. Using a simpler system would be easy to flow for busy healthcare workers. Borg (2014) discusses the Hofstede model for behavioural change with the key to improved infection control and prevention behaviour through effective education, motivation and system change [27]. By moving to something simpler it is not unsurprising that a one wipe system would be taken up more readily by staff and undertaken appropriately. The use of combination wipes fits well with a human factors approach, being available at the point of use and maximising the opportunity for correct practice. Further work is needed to elucidate the behavioural science of healthcare workers into cleaning. Although behaviour will be a factor in the observed reduction in MRSA acquisitions seen across QEHB it should be noted that the wipe QEHB changed to might be more effective. Ramm et al., (2015) demonstrated that different types of detergent wipes and different wipe substrates can be more effective at preventing the transfer of MRSA [25]. Changing to a more effective wipe could also explain the results seen in the present study. The final factor that could have contributed in a reduction of MRSA acquisitions across QEHB is training and education associated with the new wipe change. Borg (2014) discusses that education plays a role in infection prevention and control behaviour [27]. Training healthcare workers in how to use the new wipe would improve how areas within QEHB are cleaned. The numbers of nurses trained at QEHB in how to use wipe A was 1160 between April 2016- December 2017. In total there are around 2500 nurses employed within QEHB so not all staff have been trained in the use of the wipe. The effect 
of training is well documented in the literature however further work is needed to elucidate the true impact of cleaning training packages in the healthcare setting [28]. The effects of training are usually temporary however improvement trends continues after training packages have been implemented [28]. The effect of training could also in part explain the observations seen in the current report. It also must be noted that the authors did not look at the effect of trained vs non-trained nurses in the different specialities where differences in training could have been observed.

\section{Conclusion}

Strengths of this study include the use of segmented Poisson regression models which allow for changes in gradient as well as level, and so can assess the cumulative impact of any intervention more clearly [17]. Importantly the study was performed in a low prevalence setting, with a baseline rate of 20.7 MRSA acquisitions per 100,000 patients. One cannot rule out the possibility that other factors other than changing to a new detergent/disinfectant wipe may partly explain the difference seen in the rates of MRSA acquisitions across QEHB. In consideration of other variables that could have contributed to the increase in MRSA, we examined whether poor infection control procedures had a part to play. We found that there was no evidence that hand hygiene; use of personal protective equipment and environmental cleanliness had seen reductions in compliance. Audit data provided from QEHB demonstrates comparable rates during periods of the change from a two-wipe nurse led cleaning regime to a one wipe regime. The authors did not undertake any analysis of the above factors which could have contributed to the observed result. Further statistical models are needed to identify if other confounders may explain the results seen in this report. This report suggests that there is an association between the use of a one wipe regime and the reduction in spread and incidence of healthcare associated MRSA.

\section{Acknowledgements \\ We would like to thank the Infection Prevention and Control Team at the University Hospitals Birmingham NHS Foundation Trust and the clinical trainers at GAMA healthcare.}

\section{Funding}

None.

\section{Availability of data and materials}

Not applicable.

\section{Authors' contributions}

All authors have contributed to the manuscript. MW, $\mathrm{KH}$ and $\mathrm{EH}$ have helped draft the manuscript. MW has analysed all the data. The lead author MG has written and prepared the manuscript. All authors read and approved the final manuscript.

\section{Authors' information}

None.
Ethics approval and consent to participate

Not applicable

\section{Consent for publication}

Not applicable

\section{Competing interests}

MG is in receipt of a research grant from GAMA healthcare however this is independent and before this current study.

\section{Publisher's Note}

Springer Nature remains neutral with regard to jurisdictional claims in published maps and institutional affiliations.

\section{Author details}

${ }^{1}$ University Hospitals Birmingham NHS Foundation Trust, Queen Elizabeth Hospital Birmingham, Edgbaston, Birmingham B15 2WB, England. ${ }^{2}$ Institute of Microbiology and Infection, The University of Birmingham, Edgbaston, Birmingham, England. ${ }^{3}$ Gloucestershire Hospital's NHS Foundation Trust, Gloucester Royal Hospital, Great Western Road, Gloucester GL1 3NN, England.

Received: 27 July 2018 Accepted: 3 December 2018

Published online: 19 December 2018

\section{REFERENCES}

1. Garvey MI, Winfield J, Wiley C, Reid M, Cooper M. Reduction in methicillinresistant Staphylococcus aureus colonisation: impact of a screening and decolonisation programme. J Infect Prev. 2016;17(6):294-7.

2. Simor AE. Staphylococcal decolonization: an effective strategy for prevention of infection? Lancet Infect Dis. 2011;11:952-62.

3. Simor AE, Loeb M. Epidemiology of healthcare-associated Staphylococcus aureus infections. In: Crossley KB, Jefferson KK, Archer G, Fowler Jr VG, editors. Staphylococci in human disease. 2nd ed. Oxford: Blackwell Publishing; 2009. p. 290-309.

4. Robotham JV, Graves N, Cookson BD, et al. Screening, isolation, and decolonization strategies in the control of meticillin resistant Staphylococcus aureus in intensive care units: cost effectiveness evaluation. BMJ. 2011; 5(343): $\mathrm{d} 5694$.

5. Baldwin NS, Gilpin DF, Tunney MM, et al. Cluster randomised controlled trial of an infection control education and training intervention programme focusing on methicillin-resistant Staphylococcus aureus in nursing homes for older people. J Hosp Infect. 2010;76:36-41.

6. Robicsek A, Beaumont JL, Thomson RB Jr, Govindarajan G, Peterson LR. Topical therapy for meticillin-resistant Staphylococcus aureus colonization: impact on infection risk. Infect Control Hosp Epidemiol. 2009;30:623-32.

7. Coia JE, Duckworth GJ, Edwards DI, et al. Joint Working Party of the British Society of Antimicrobial Chemotherapy; Hospital Infection Society; Infection Control Nurses Association. Guidelines for the control and prevention of meticillin-resistant Staphylococcus aureus (MRSA) in healthcare facilities. J Hosp Infect. 2006;63:S1-44.

8. Bradley CW, Wilkinson MA, Garvey MI. The effect of universal decolonisation with screening in critical care to reduce MRSA across an entire hospital. Infect Control Hosp Epidemiol. 2017;38(4):430-5.

9. Dancer SJ. Controlling hospital-acquired infection: focus on the role of the environment and new technologies for decontamination. Clin Microbiol Rev. 2014;27(4):665-90.

10. Vickery K, Deva A, Jacombs A, Alan J, Valente P, Gosbell IB. Presence of biofilm containing viable multiresistant organisms despite terminal cleaning on clinical surfaces in an intensive care unit. J Hosp Infect. 2012;80(1):52-5.

11. Mitchell BG, Dancer SJ, Anderson M, Dehn E. Risk of organism acquisition from prior room occupants: a systematic review and meta-analysis. J Hosp Infect. 2015;91:35-44.

12. Hardy KJ, Gossain S, Henderson N, et al. Rapid recontamination with MRSA of the environment of an intensive care unit after decontamination with hydrogen peroxide vapour. J Hosp Infect. 2007;66:360-8.

13. Garvey MI, Bradley CW, Holden K, Oppenheim B. Outbreak of colonal complex 22 Panton-Valentine leucocidin-positive meticillin-resistant Staphylococcus aureus. J Infect Prev. 2017;18(5):224-30.

14. Garvey MI, Pichon B, Bradley CW, Moiemen NS, Oppenheim B, Kearns AM. Improved understanding of an outbreak of methicillin resistant 
Staphylococcus aureus in a regional burns centre via whole-genome sequencing. J Hosp Infect. 2016;94:401-4.

15. Kaufmann ME. Pulsed-field gel electrophoresis. In: Woodford N, Johnson AP, editors. Methods in molecular medicine 15: molecular bacteriology: protocols and clinical applications. New Jersey: Humana Press; 1998. p. 33-50.

16. Guidance on the reporting and monitoring arrangements and post infection review process for MRSA bloodstream infections from. Patient Safety Domain. In: NHS England; 2014. Available at: https://www.england. nhs.uk/patientsafety/wp-content/uploads/sites/32/2014/02/post-infguidance2.pdf.

17. Gebski V, Ellingson K, Edwards J, Jernigan J, Kleinbaum D. Modelling interrupted time series to evaluate prevention and control of infection in healthcare. Epidemiol. Infect. 2012;140:2131-41.

18. R version 3.4.3 (2017-11-30) - "Kite-Eating Tree". The R Foundation for Statistical Computing

19. Robotham JV, Deeny SR, Fuller C, Hopkins S, Cookson B, Stone S. Costeffectiveness of national mandatory screening of all admissions to English National Health Service hospitals for meticillin-resistant Staphylococcus aureus: a mathematical modelling study. Lancet Infect Dis. 2016;16:348-56.

20. Fuller C, Robotham J, Savage J, et al. The National One Week Prevalence Audit of Universal Meticillin-Resistant Staphylococcus aureus (MRSA) Admission Screening 2012. PLoS one. 2013;12:e74219.

21. Royal College of Nursing. Wipe it out, one chance to get it right. Essential practice for infection prevention and control. Guidance for nursing. 2014. https://www.rcn.org.uk/professional-development/publications/pub-003876 last accessed 10th May 2018

22. Health Protection Scotland. Literature review and practice recommendations: existing and emerging technologies used for decontamination of healthcare environment. 2017. http://www.hps.scot.nhs. uk/resourcedocument.aspx?id=5957

23. Kundrapu S, Sunkesula VC, Jury LA, Sitzlar BM, Donskey CJ. Daily disinfection of high-touch surfaces in isolation rooms to reduce contamination of healthcare workers hands. Infect Control Hosp Epidemiol. 2012;33(10):1039-42.

24. Dancer SJ. Missing a trick? Response to: 'Disinfectant wipes are appropriate to control microbial bioburden from surfaces'. J Hosp Infect. 2016;92:204-11.

25. Ramm L, Siani H, Wesgate R, Maillard JY. Pathogen transfer and high variability in pathogen removal by detergent wipes. Am J Infect Control. 2015;43(7):724-8.

26. Sattar SA, Bradley C, Kibbee $R$, et al. Disinfectant wipes are appropriate to control microbial burden from surfaces: use of a new ASTM standard test protocol to demonstrate efficacy. J Hosp Infect. 2015;91(4):319-25.

27. Borg MA. Lowbury Lecture 2013. Cultural determinants of infection control behaviour: understanding drivers and implementing effective change. J Hosp Infect. 2014;86(3):161-8.

28. Brusaferro S, Arnoldo L, Cattani G, et al. Harmonizing and supporting infection control training in Europe. J Hosp Infect. 2015;89:351-6.

Ready to submit your research? Choose BMC and benefit from:

- fast, convenient online submission

- thorough peer review by experienced researchers in your field

- rapid publication on acceptance

- support for research data, including large and complex data types

- gold Open Access which fosters wider collaboration and increased citations

- maximum visibility for your research: over $100 \mathrm{M}$ website views per year

At BMC, research is always in progress.

Learn more biomedcentral.com/submissions 